\title{
Li-rod based muon ionization cooling channel
}

\author{
T. Zolkin* \\ The University of Chicago, Department of Physics, KPTC 201, 5720 South Ellis Avenue, Chicago, Illinois 60637-1434, USA
}

\author{
A. N. Skrinsky \\ Budker Institute of Nuclear Physics, 11 akademika Lavrentieva prospect, Novosibirsk 630090, Russia
}

(Received 1 September 2011; published 12 April 2012)

\begin{abstract}
A muon ionization cooling channel based on lithium rods (Li-rod) has been under consideration since the middle of the 1980s. Features of muon beam motion in such a channel are discussed, namely, an influence of nonparaxiality of motion and transverse-longitudinal coupling. Most simulations of muon beam cooling were performed using the specially developed software LYRICS (lithium rod ionization cooling simulation); a comparison between its results and the predictions of a linear model serves both to examine the simulation code and to determine the contribution of nonparaxiality to the beam motion. For numerical examples, we used muons around $200 \mathrm{MeV}$ total energy since such energy is close to optimal. The idea of the inclusion of a symplectic (nondissipative) emittance exchanger to the cooling channel, which allows one to cool in all degrees of freedom, is introduced. The appropriate beam parameters for emittance exchange procedure and their dependence on transverse emittance and beam longitudinal parameters are discussed.
\end{abstract}

DOI: 10.1103/PhysRevSTAB.15.043501

PACS numbers: 41.75.Lx, 05.10.Gg, 05.10.Ln, 07.05.Tp

\section{INTRODUCTION}

\section{A. General overview}

One of the most important steps that enabled progress in high energy physics was implementation of colliding beams over 50 years ago. Higher energy and higher productivity (luminosity) of colliders are important for any further progress in HEP.

A proton-proton collision at very high energy is equivalent to a collision of its fundamental constituents with an effective energy of about $1 / 6$ of the initial proton energy, though with a very wide energy spectrum. As a result of this complex collision, analysis of experimental data and extraction of properties of fundamental interactions becomes more difficult than for elementary particles.

Therefore, it would be highly beneficial to study high energy processes in collisions of elementary particles. For center-of-mass energy up to about $1 \mathrm{TeV}$ linear electronpositron colliders could solve the problem acceptably well. However, in order to reach sufficiently high luminosity, high intensity bunches are required. Such high-density $e^{-}$ $\left(e^{+}\right)$bunches produce very high focusing fields, so primary particles emit too many photons. The effect can be substantially reduced with the use of wide but very thin bunches of the same transverse cross section. However, even in this case, the resulted energy spread reaches several

\footnotetext{
*zolkin@uchicago.edu

†skrinsky@inp.nsk.su
}

Published by the American Physical Society under the terms of the Creative Commons Attribution 3.0 License. Further distribution of this work must maintain attribution to the author(s) and the published article's title, journal citation, and DOI. tens of percent for $1 \mathrm{TeV}$ beams. The use of electronelectron collisions instead leads to strong repulsion, which results in orders of magnitude lower luminosity.

The source of this problem is in smallness of the electron mass, as the emitting power in a collision is proportional to $E^{2} / m^{4}$. The effect can be practically suppressed if heavier leptons are used. The only heavier lepton with acceptably long lifetime is a muon whose mass is a factor of 200 higher than that of an electron (the idea of a muon collider was proposed by Budker [1,2] in 1969 and later described in more detail by Skrinsky [3] in 1971). The muon lifetime is $2.2 \mu \mathrm{s}$ in its rest frame and rises proportionally to its total energy in the laboratory frame. As a result, one can expect up to 1000 bunch collisions in a high-field cyclic collider prior to muon decay. Thus, in a collider of a given perimeter and guiding magnetic field, use of muons instead of protons allows one to study fundamental interactions at 6 times higher energy and under much cleaner conditions (initial particles are well determined and effective monochromaticity of collisions is much, much better). That is why muon beams present a unique opportunity for a precision study of fundamental interactions at a several $\mathrm{TeV}$ center-of-mass energy scale.

However, several key technologies for a muon collider are not yet developed. First of all, it is necessary to reduce the 6-dimensional muon emittance by 5-6 orders of magnitude, preserving the beams intensity. That strong muon cooling is a must to reach sufficient luminosity.

The most promising method for muon beam cooling is to use ionization energy losses in some dense matter with a consequent compensation of lost energy (and longitudinal momentum) via the rf field. Pioneering works on ionization cooling were done by Kolomenskii [4] in 1965 and later developed by a number of authors [5-7]. Much effort is being invested to develop and study various cooling 


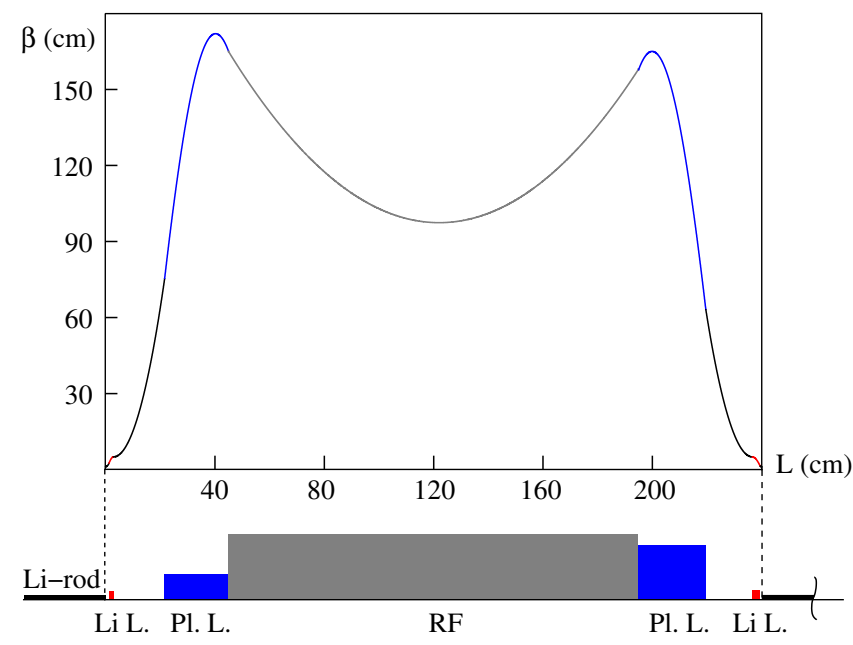

FIG. 1. Example of the beta-function behavior in one period of the cooling channel.

schemes both analytically and using computer simulation during the past 10 years (a list of the most recent references can be found in review articles by Shiltsev [8] and Zisman [9]). Recently, experimental study of some cooling techniques has been done at Fermilab MuCool Test Area [10] and at the Muon Ionisation Cooling Experiment at Rutherford Appleton Laboratory [11].

\section{B. Article structure}

This article covers more carefully the study of a cooling channel based on liquid-lithium rods (Li-rod). The main parameters of this cooling scheme are presented in Sec. II. The rods provide muon energy losses due to ionization and simultaneously strong transverse focusing by carrying a very high current along the rod. While its technical realization is still in development, Li-rod may provide sufficiently strong focusing for the final cooling stage. Different engineering aspects and technical limitations for Li-rods, as well as a list of references, can be found in [12-14]. In simulations, we used Li-rods with current up to $1 \mathrm{MA}$ and magnetic field on the surface up to $200 \mathrm{kG}$.

Predominantly, this article focuses on a muon motion analysis in a single Li-rod, which is a core of this cooling scheme, and not on a whole beam line. Different features of transverse and longitudinal muon beam motion through the rod are presented in Secs. IV and V, respectively (such as nonparaxiality and transverse-longitudinal coupling). The comparison between different simulations and linear model predictions are presented. As will be shown, the nonparaxiality of motion results in a higher rate of heating of the longitudinal degree of freedom in comparison with predictions of a paraxial model (up to 2 times). An additional rf cavity, which rotates a beam in the $\left(c d t, d E / E_{\mathrm{eq}}\right)$ plane by an angle of pi, can be placed after a main one to suppress this undesirable effect.

The idea of the inclusion of a nondissipative emittance exchanger in the cooling channel, which can upgrade the cooling to all degrees of freedom, is introduced [15]. Optimal parameters for the emittance exchange procedure are determined in Sec. VI. More complete consideration of emittance exchange procedure will be presented in future work.

At the end of this paper, one can find two appendixes where the linear models for transverse and longitudinal motion are investigated in detail.

\section{COOLING SCHEME UNDER CONSIDERATION}

The proposed cooling channel is based on Li-rods alternating with rf cavities. The matching between main elements is realized by a cascade of lithium and plasma lenses [16,17]: the short lithium lenses (strong in comparison with plasma ones) make the beta function several times larger at the exit of lithium rods (smaller at the entrance) and weaken the lowaberration functioning of plasma lenses, which have longer focal lengths. An example of parameters for one period of this cooling scheme along with beta-function behavior are presented in Table I and Fig. 1, respectively.

TABLE I. Example of element parameters for one period of the cooling channel.

\begin{tabular}{|c|c|c|c|c|c|c|c|c|c|c|c|c|}
\hline & & Li-rod & Drift & Li lens & Drift & Plasma lens & rf cavity & Plasma lens & Drift & Li lens & Drift & Li-rod \\
\hline$E^{\mathrm{a}}$ & $(\mathrm{MeV})$ & 190 & 190 & 190 & 190 & 190 & 220 & 220 & 220 & 220 & 220 & 190 \\
\hline$\beta^{\mathrm{b}}$ & $(\mathrm{cm})$ & 1.11 & 2.52 & 5 & 75.28 & 165 & 158 & 63.19 & 5 & 1.39 & 1.1 & 1 \\
\hline$H^{\mathrm{c}}$ & $(\mathrm{kG})$ & 200 & $\ldots$ & 147 & $\ldots$ & 3.1 & $\ldots$ & 7.9 & $\ldots$ & 139 & $\ldots$ & 200 \\
\hline$I^{\mathrm{d}}$ & (MA) & 0.5 & $\ldots$ & 0.78 & $\ldots$ & 0.05 & $\ldots$ & 0.26 & $\ldots$ & 0.81 & $\ldots$ & 0.4 \\
\hline$L^{\mathrm{e}}$ & $(\mathrm{cm})$ & 30 & 1.25 & 1.63 & 18.7 & 23.37 & 150 & 24.6 & 17.05 & 2.84 & 0.56 & 30 \\
\hline$D^{\mathrm{f}}$ & $(\mathrm{cm})$ & 1 & $\ldots$ & 2.14 & $\cdots$ & 6.3 & $\ldots$ & 13.32 & $\ldots$ & 2.34 & $\ldots$ & 0.8 \\
\hline
\end{tabular}

${ }^{a}$ Particle full energy at the exit of element.

${ }^{\mathrm{b}}$ Beta function at the exit of element.

${ }^{c}$ Magnetic field on the surface of element.

${ }^{\mathrm{d}}$ Current through the element.

${ }^{\mathrm{e}}$ Length of element.

${ }^{\mathrm{f}}$ Diameter of element. 


\section{BEAM MOTION SIMULATION}

The multipurpose software "lithium rod ionization cooling simulation" (LYRICS) has been developed for a study of a scheme of final cooling for muon beams based on consequent lithium rods. It can simulate the 6-dimensional motion of a muon beam through the matter including such effects as nonparaxiality, dissipation, and stochastic processes like scattering or fluctuations of energy losses. The fluctuation of energy losses is considered as a Gaussian stochastic process, since Li-rod corresponds to a thick absorber; in this limit Gaussian approximation works with high precision [18]. Also LYRICS allows simulation of the motion in matching sections, including acceleration in rf cavities (with taking into account transverse focusing due to transverse components of rf fields).

\section{TRANSVERSE MOTION}

In the paraxial case, the evolution of the second moments of the beam $\left\langle x^{2}\right\rangle,\left\langle x^{\prime 2}\right\rangle,\left\langle x x^{\prime}\right\rangle$ and the transverse emittance $\varepsilon_{\text {tr }}$ is sufficiently simple and can be described analytically (see Appendix A). Examples of their behavior are presented in Fig. 2. Each of these quantities tends to its asymptotic from above (below) if its initial value is bigger (smaller) than the equilibrium one [see Appendix A, Eq. (A4)]. In the most general case, when the initial values of the second moments are unmatched with the optical functions of the cooling channel, they are damped with oscillations (grey lines on the graph). Despite the fact that in both cases the cooling rate is the same, there is no doubt that these oscillations are undesirable because they can

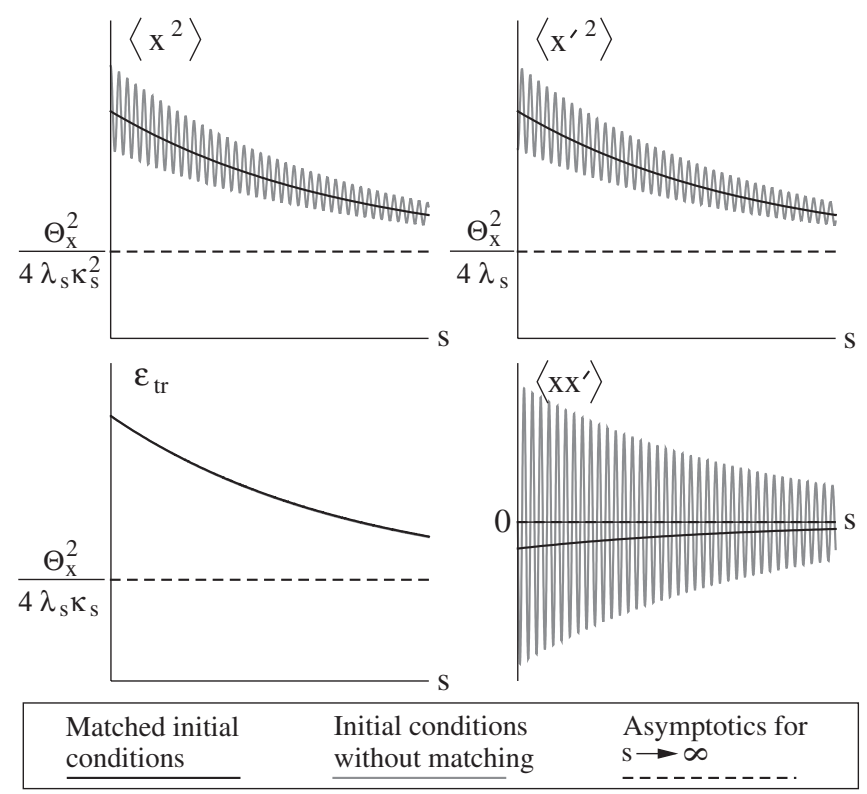

FIG. 2. Stylistic impression of the behavior of the second moments of the beam and transverse emittance due to ionization cooling in the cases of matched initial conditions (black curves) and unmatched ones (gray curves). lead to additional particle losses on the physical aperture and should be avoided [see Appendix A, Eq. (A6)].

\section{A. Comparison of linear model and simulation}

A comparison between the simulation results and the predictions of a paraxial linear model is presented below, with specific examples shown in Fig. 3 for two different values of the initial transverse emittance. The simulation was made for ten consecutive lithium rods similar to that considered in Sec. II. Each of them has a length of $30 \mathrm{~cm}$ and a field on its surface of $200 \mathrm{kG}$. The diameter of rods was chosen as 5 and $2 \mathrm{~cm}$ for the (a) and (b) cases, respectively. Particle motion in the matching and acceleration sections were simulated as ideal thin transformations such that the full energy of a beam, $\gamma_{\mu} m_{\mu} c^{2}$, at the entrance to each rod is equal to $220 \mathrm{MeV}$ and the second moments are matched with the channel optics. The beam energy used in the linear model is about $205 \mathrm{MeV}$, corresponding to the simulated beam energy. It turns out that this relatively simple analytical model agrees with simulation to high precision, with a discrepancy of less than $5 \%$.

To determine in detail how longitudinal motion affects transverse cooling, beam passage through a single rod was examined for multiple values of initial longitudinal emittance, or more exactly, the initial position of the beam on the $\left[\left\langle(c \Delta t)^{2}\right\rangle_{0}^{1 / 2},\left\langle\left(\Delta E / E_{\text {eq }}\right)^{2}\right\rangle_{0}^{1 / 2}\right]$ plane, with a fixed initial transverse emittance. It has been found that only in cases of relatively small transverse emittance (close to the equilibrium value) or very large initial energy spread (more than $10 \%$ ) is there an around 5\% decrease in the rate of cooling compared to the linear model. This confirms that the influence of longitudinal motion on the transverse cooling is indeed small.

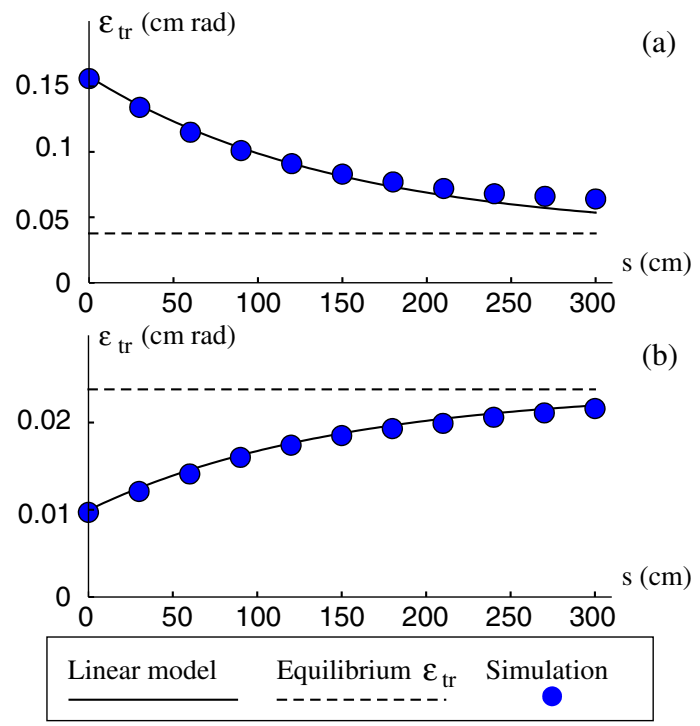

FIG. 3. Linear model of transverse motion compared to simulation in the cases of (a) large initial transverse emittance and (b) small one. 


\section{LONGITUDINAL MOTION}

Similar to the transverse motion, the longitudinal motion of the beam in matter can be described analytically by a linear approach (see Appendix B). The heating of longitudinal moments occurs by two processes: antidamping under the action of ionization friction and "diffusion"a fluctuation of ionization losses.

In this model the behavior of the root-mean-square energy spread [Eq. (B5a)] can be described using a certain characteristic value

$$
\Delta_{\mathrm{ch}}=\sqrt{\Theta_{d E / E}^{2} / 4 \lambda_{t}}
$$

If the initial energy spread in a beam,

$$
\Delta \equiv \sqrt{\left\langle\left(\Delta E / E_{\mathrm{eq}}\right)^{2}\right\rangle_{0}}
$$

is larger than this value, an exponential growth of the rootmean-square energy spread by antidamping is observed. Vice versa, for the case when $\Delta<\Delta_{\text {ch }}$, this longitudinal second moment has an extremely fast growth described by diffusion initially (the smaller the initial value of the energy spread, the faster the growth), which becomes exponential. The similar quantity $\sqrt{\Theta_{x}^{2} / 4 \lambda_{s}}$, for the case of the transverse motion, refers to the equilibrium angular spread determined by the competition of damping and diffusion processes. Numerical examples of the evolution of the root-mean-square energy spread is presented in Fig. 4 (top left plot), where the average full energy is chosen as $205 \mathrm{MeV}$.

In contrast to mean square energy spread, the behavior of the mean square spread of the arrival time, $\left\langle(c \Delta t)^{2}\right\rangle$, is determined by a function of all longitudinal initial data [see Eq. (B5c)]. But it has the asymptotics which depends on $\Delta$ only, regardless of the initial value $\delta \equiv \sqrt{\left\langle(c \Delta t)^{2}\right\rangle_{0}}$ :

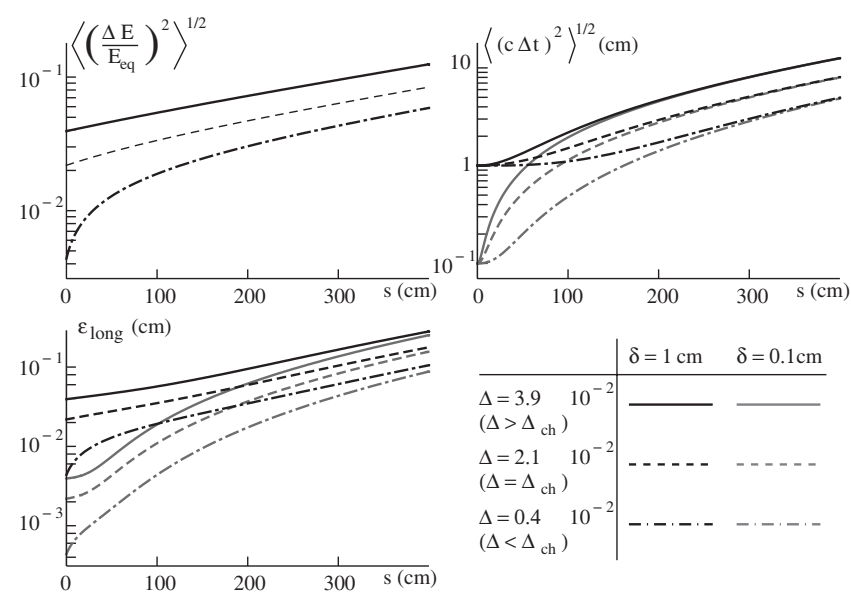

FIG. 4. Evolution in matter of the longitudinal second moments $\left\langle(c \Delta t)^{2}\right\rangle^{1 / 2}$ and $\left\langle\left(\Delta E / E_{\mathrm{eq}}\right)^{2}\right\rangle^{1 / 2}$ as well as the longitudinal emittance $\varepsilon_{\text {long }}$ for different initial values.

$$
\lim _{t \rightarrow \infty}\left\langle(c \Delta t)^{2}\right\rangle=\left(\Delta^{2}+\frac{\Theta_{d E / E}^{2}}{4 \lambda_{t}}\right)\left(\frac{c}{\beta_{\mathrm{eq}}^{2} \gamma_{\mathrm{eq}}^{2}} \frac{e^{2 \lambda_{t} t}}{2 \lambda_{t}}\right)^{2}
$$

Several examples of the behavior of root-mean-square spread of the arrival time are shown in Fig. 4 (top right) for different values of $\Delta$ and $\delta$ [the value of $\langle(c \Delta t) \times$ $\left.\left(\Delta E / E_{\mathrm{eq}}\right)\right\rangle_{0}$ is equal to zero for simplicity for all cases].

The evolution of the longitudinal emittance is given at the bottom of this figure for all considered sets of initial beam second moments. It shows that, even for the same initial values of $\varepsilon_{\text {long }}$, its behavior depends on the relation between $\delta$ and $\Delta$ (compare gray solid and black dot-dashed curves). Regardless of the fact that in matter both second moments grow $\propto e^{4 \lambda_{t} t}$ for the large time asymptotic, longitudinal emittance grows $\propto e^{2 \lambda_{t} t} \sqrt{\lambda_{t} t}$.

\section{A. Comparison of linear model and simulation}

The same analysis of beam passage through one rod with variation of the initial conditions (such as initial $\varepsilon_{\text {tr }}$ and the initial beam position on the $(\delta, \Delta)$ plane), which has been used in Sec. IVA, is also useful for describing the dependence of longitudinal second moments heating on transverse beam parameters.

The growth of $\left\langle\left(\Delta E / E_{\text {eq }}\right)^{2}\right\rangle^{1 / 2}$ (the ratio of the final rootmean-square energy spread upon the exit of a lithium rod to that at the entrance) is shown in Fig. 5(a). As expected, it is independent of the transverse beam parameters, even for large values (though undoubtedly these parameters are limited from above to values reasonable for this cooling scheme).

In contrast, the growth of $\left\langle(c \Delta t)^{2}\right\rangle^{1 / 2}$ conforms to the linear model prediction with confidence only in the case of small transverse emittance (lines with circle symbols in Fig. 5(b)]; the increment of the spread in the arrival time upon the exit of the rod grows with the transverse emittance (at fixed initial parameters, namely, $\delta$ and $\Delta$ ). This too is a result of nonparaxiality of motion-the presence of particles with big angles entails a significant increase in the paths they take, relative the equilibrium one. This effect is demonstrated for two cases with different initial energy spreads (black and gray solid lines which refer to large and small values of $\Delta$, respectively).

\section{B. "Head-tail" rotation}

Upon studying the cooling process as a whole, simulations show that due to nonparaxiality the longitudinal emittance grows faster than the linear model predicts (up to 2 times).

A study of the distribution of particle arrival times during the cooling process reveals its deformation after passing through a few rods (a numerical example is presented in Fig. 6, top left). There are three main differences between the initial and final distributions (black and white bars, respectively). First of all, the packet spreads faster 

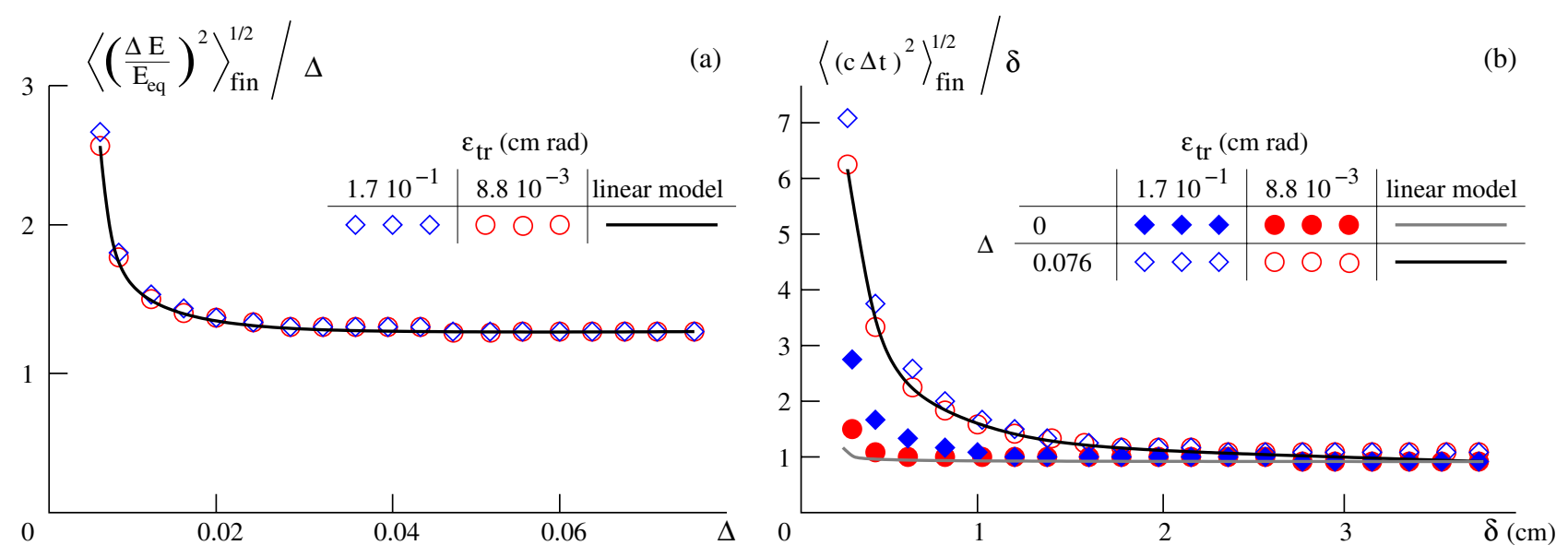

FIG. 5. Growth of longitudinal second moments of a beam after a single rod passage under different initial conditions in comparison with a linear model.

than predicted by the linear model. Also, a long tail forms asymmetrically as a result of lagging particles. The third difference, which is a direct result of the emergence of a long tail, is that the "center of gravity" of the distribution shifts from the peak position. All this means that nonparaxial particles lose more energy than those at the center, causing them to drop behind the main part of the beam. This emphasizes again the importance of taking transverse parameters into account during a study of longitudinal motion.

A similar analysis of the distribution of the relative energy (Fig. 6, top right) also shows particles with bigger losses, but the amount of distribution deformation is not as significant in this case.

It appears that a procedure of a beam symmetrization ("head-tail" rotation [17]), consisting of beam rotation in longitudinal phase space by an angle of $\pi$, can completely solve this problem; it allows one to obtain the same rate of

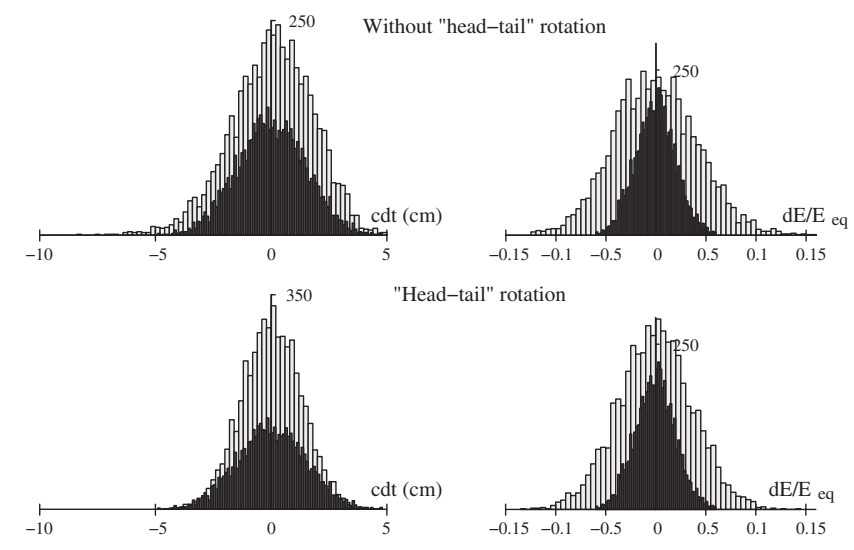

FIG. 6. The evolution of the distribution of the arrival time and the relative energy after passing through 5 rods in cases of headtail rotation usage and without it; $\square$-initial distribution; $\square$ distribution after passing 5 rods. longitudinal emittance growth in simulation as the linear model predicts without additional increase due to nonparaxiality effects. Similar simulations of the evolution of $c d t$ and $d E / E_{\text {eq }}$ distributions but with head-tail rotation usage are shown in the bottom of Fig. 6. Even just visually, the final distributions are much more symmetric and narrow. The beam symmetrization was simulated as a thin ideal process after the second and fourth rods. Practically, it can be realized by an additional rf section with a zero acceleration gradient placed in each period of the cooling system (or performing an additional rf gymnastic in sections of energy recovery). The influence of this procedure on a muon's lifetime and its technical parameters will be estimated in future work.

\section{EXTENSION TO 6D COOLING}

The redistribution of the cooling decrement between longitudinal and transverse degrees of freedom is the general idea behind the organization of $6 \mathrm{D}$ cooling or the slowing down of the longitudinal emittance heating for muons (for details, see, e.g. [19]). A typical beam line with such a redistribution should include magnetic elements with a gradient (e.g. dipoles) to provide dispersion, and additional "wedge" absorbers to organize momentumdependent energy loss (ideally placed at the location of the maximum of dispersion). The helical cooling channel concept, reverse emittance exchange, wedge absorber, or continuous gaseous absorber schemes are just a few examples. However, analytical calculations show that the density gradient in the Li-rod helix is not enough for cooling in all degrees of freedom, while a magnetic field in a system is limited from above by a practical value.

The usage of the symplectic emittance exchange can offer an alternative scenario. The linear transformation, $\mathcal{R}$, is said to be symplectic if it satisfies $\mathcal{R}^{T} J \mathcal{R}=J$, where $J$ is a fixed nonsingular skew-symmetric matrix 


$$
J=\left[\begin{array}{cc}
0 & I_{n} \\
-I_{n} & 0
\end{array}\right]
$$

$I_{n}$ is the $n \times n$ identity matrix, and $2 n$ is a dimension of a square matrix of transformation $\mathcal{R}$. In contrast to the idea of decrement redistribution, symplectic emittance exchange does not require the employment of dissipative forces; moreover, the transformation is linear and therefore preserves phase space because of Liouville's theorem. Note that for the fully uncoupled and uncorrelated beam, as best, one can swap two eigenemittances (while keeping the third one fixed) if beam will remain uncoupled or mix all of them together by means of projected emittances, since under any linear Hamiltonian transformation the eigenemittances of a beam are invariant up to a reordering (for details, see, e.g. [20,21]). Therefore one can employ the emittance exchange to redistribute phase-space volume from longitudinal to one of the transverse degrees of freedom (as soon as longitudinal emittance becomes too large to operate with it) to continue the cooling process.

One of the possible technical realizations of the emittance exchange between the longitudinal and one of the transverse degrees of freedom is a combination of a four dipole-magnet chicane and a dipole-mode cavity placed at the dispersive region of chicane [20]. Here we do not focus on any exact implementation of an emittance exchanger but will consider it as a thin ideal process in simulations of a cooling. Some general limitations for exchange usage will be discussed in Sec. VI A.

Note that neither the redistribution of the cooling decrement nor the symplectic emittance exchange can change the total 6D damping rate (analogous with Robinson's theorem).

\section{A. Limitations for emittance exchange usage}

The final value of full emittance is determined by the equilibrium value of the transverse emittance [Eq. (A7)] and how much phase-space volume is redistributed from the longitudinal to transverse degrees of freedom during the cooling. Therefore, the wish to redistribute as much as possible is natural, but there is a certain limitation: if the longitudinal second moment values are too small, the diffusion (a fluctuation of ionization losses) begins dominating and longitudinal heating goes faster, which can even lead to full 6D emittance heating. In Fig. 7 two sketches of cooling with redistribution demonstrate how an excessive redistribution (bottom row of figures) can slow down 6D cooling. Therefore, a proper value for the longitudinal emittance after redistribution and the dependence of that value on the transverse motion should also be determined.

By scanning the $(\delta, \Delta)$ plane of initial beam parameters, one can determine the region of parameters optimal for cooling as a function of the transverse phase-space (for a certain length of rod). An example of such a scan is presented in Fig. 8 for the $30 \mathrm{~cm}$ rod with a $2 \times 10^{5}$ Gauss limitation for the field on its surface. The gray color
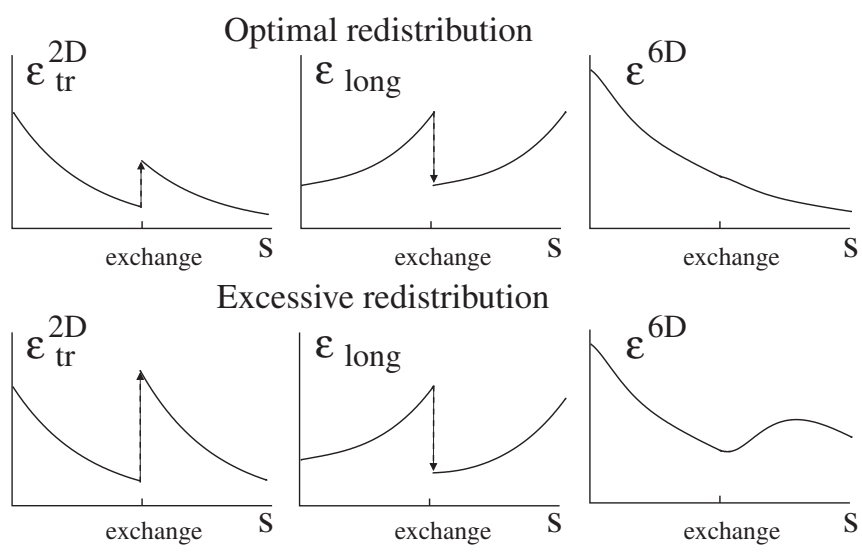

FIG. 7. Stylistic impression of the behavior of 2D transverse (involved into the redistribution process), longitudinal, and 6D emittances as functions of the length passed in matter during cooling. Top and bottom rows of figures correspond to the optimal redistribution from the longitudinal to transverse degree of freedom and excessive redistribution with undesirable full emittance increment, respectively. The redistribution is shown as a thin ideal process by dashed arrow.

corresponds to the area of initial parameters where the antidamping dominates over diffusion. The " 0 " emittance case refers to a simulation where transverse motion was completely removed and, therefore, the obtained region is very close to that predicted by the linear model. One can therefore conclude that, while transverse motion is relatively independent of longitudinal parameters, longitudinal motion is strongly influenced by transverse beam parameters.

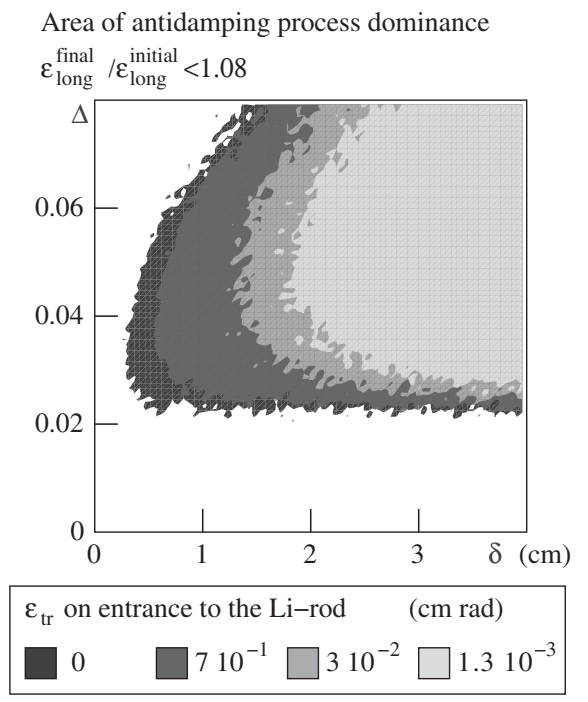

FIG. 8. The region of longitudinal parameters $\delta$ and $\Delta$ in which the antidamping process dominates over the fluctuation of ionization losses (in other words, the region where the growth of the longitudinal emittance $\left(\varepsilon_{\text {long }}^{\text {fin }} / \varepsilon_{\text {long }}^{\text {ini }}\right)$ is below a preassigned value defined from the asymptotics) after passage through one lithium rod for different values of the initial transverse emittance. 


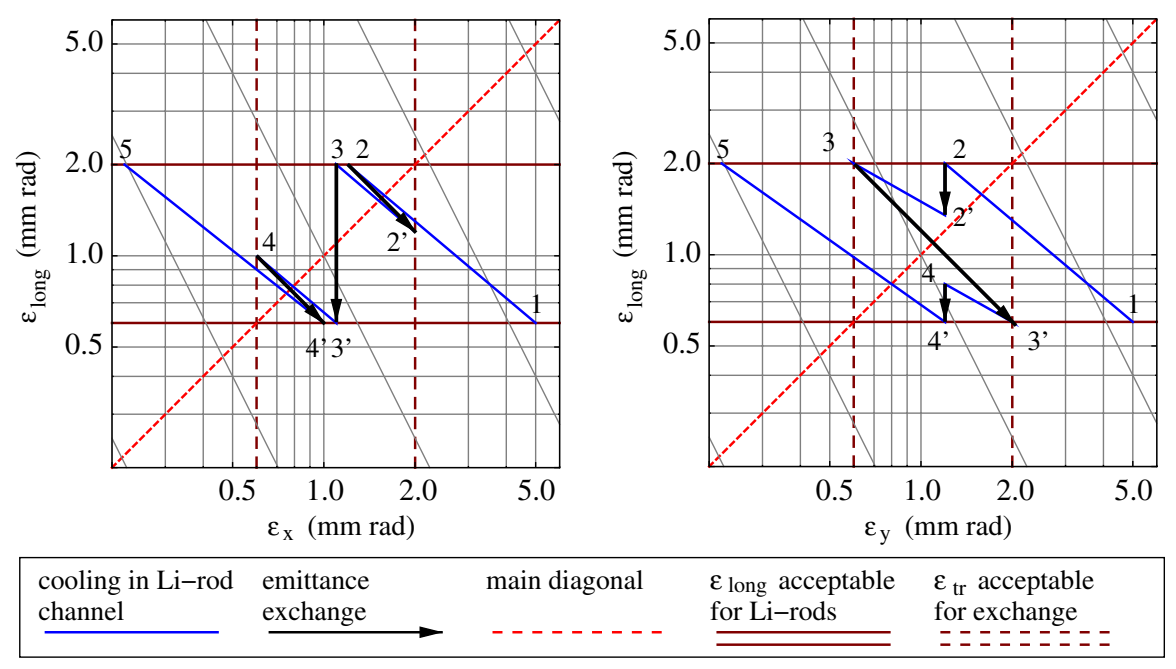

FIG. 9. Conceptual view of emittance exchange usage for Li-rod cooling scheme. The consecutive steps are as follows: beam cooling in the linear Li-rod based channel $(1 \rightarrow 2)$, symplectic emittance exchange from horizontal $(x)$ to longitudinal degree of freedom $\left(2 \rightarrow 2^{\prime}\right)$, second Li-rod based cooling channel $\left(2^{\prime} \rightarrow 3\right)$, symplectic emittance exchange from vertical $(y)$ to longitudinal degree of freedom $\left(3 \rightarrow 3^{\prime}\right)$, third Li-rod based cooling channel $\left(3^{\prime} \rightarrow 4\right)$, symplectic emittance exchange from horizontal $(x)$ to longitudinal degree of freedom $\left(4 \rightarrow 4^{\prime}\right)$, final Li-rod based cooling channel $\left(4^{\prime} \rightarrow 5\right)$.

\section{B. Preliminary simulation with thin exchange}

A conceptual view of emittance exchange usage is presented in Fig. 9. Since the cooling process is not symmetric with respect to transverse coordinates $x$ and $y$, there are two plots $\varepsilon_{\text {long }}$ vs $\varepsilon_{x}$ and $\varepsilon_{\text {long }}$ vs $\varepsilon_{y}$. Three emittance exchanges are used as follows: Long with $\mathrm{X}$, Long with $\mathrm{Y}$, and then Long with $\mathrm{X}$ again. Diagonal gray lines are the levels of a full 6D emittance for the case of equal transverse emittances. The brown horizontal lines approximately show the range of longitudinal parameters where
Li-rods can be used: the bottom bound refers to a diffusiondominance region which was described above, and the top bound refers to the rf-system parameters. The symplectic emittance exchange lets one map a point on this plot relative to a main diagonal (red dashed line). Therefore, the intersection between horizontal band and its flip image (brown dashed lines) is an area where emittance exchange procedure can be used. Otherwise, after the exchange the longitudinal emittance will be out of range of an adequate for this scheme parameters.
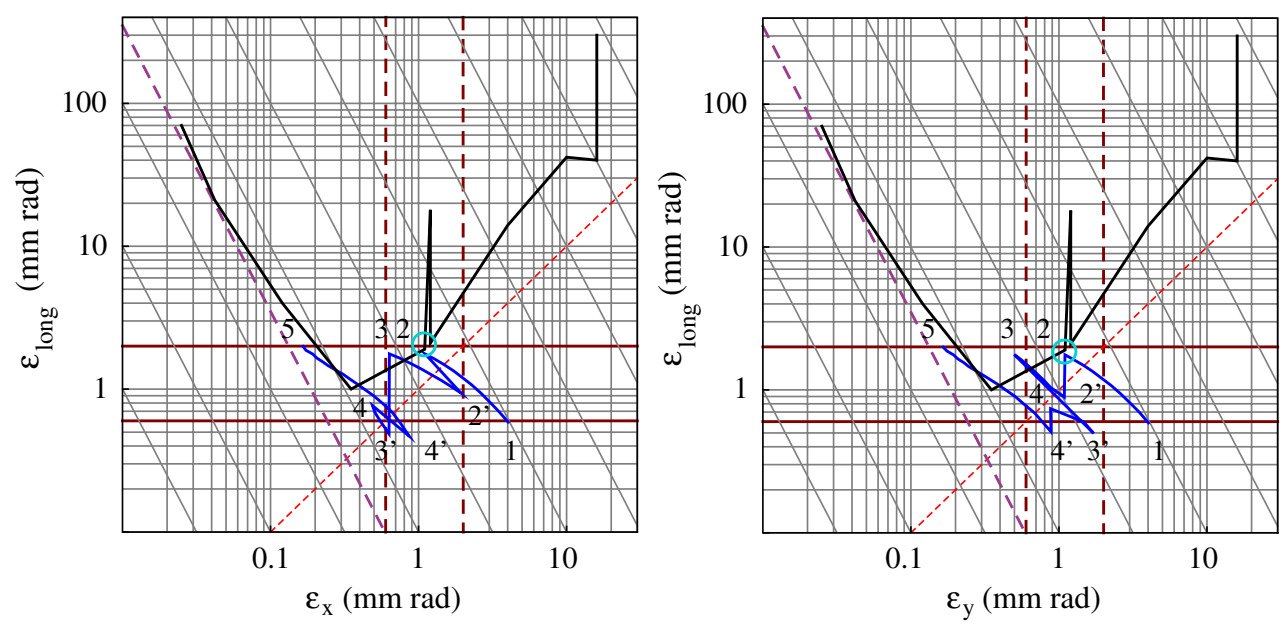

\begin{tabular}{|lll|}
\hline $\begin{array}{l}\text { cooling in Li-rod channel } \\
\text { with emittance exchange }\end{array}$ & $\begin{array}{l}\text { general cooling } \\
\text { scenario }\end{array}$ & $\begin{array}{l}\text { lowest achievable full emittance } \\
\text { for the general scenario } \\
-\end{array}$
\end{tabular}

FIG. 10. Preliminary simulation of cooling with emittance exchange usage (blue line) in comparison with general cooling scenario for a muon collider (black line). The simulation is made in accordance with the conceptual view presented in Fig. 9, where steps of cooling are described. 
The simulation of this idea is presented in Fig. 10. The emittance exchange, rf, and matching were simulated as ideal thin processes, but all Li-rods were simulated with taking into account all main effects such as dissipation, scattering, and energy fluctuation. The black line is a general scenario of a full cooling process for muon collider considered in many articles (e.g. [8]).

\section{SUMMARY}

The motion of a muon beam in the final cooling scheme based on lithium rods has been simulated using LYRICS software. The analytical linearized model of muon beam motion, which helps to check the code of the developed software, was used to determine the influence of nonparaxiality and the interdependence of transverse and longitudinal motion. Preliminary estimations of the optimal longitudinal beam parameters for the symplectic emittance exchange procedure, which will possibly help to organize the cooling of all degrees of freedom, are presented in Sec. VI A.

The main conclusions are: (i) The selected linear model is able to describe the transverse motion with high precision independent of beam longitudinal parameters (in the range reasonable for all parameters in this type of cooling). (ii) The evolution of the relative energy deviation also converges with the linear model for a wide range of transverse emittances and is independent of the deviation in the arrival time. In contrast, the spread of the arrival time agrees with the linear model only in the case of small transverse emittance, which is a direct consequence of nonparaxiality (particles with large angular deviations follow a longer path in matter than the equilibrium one, which results in the "tail" formation in the direction of delayed particles). Also, the dependence of the root-mean-square arrival time on the transverse emittance makes the optimal beam longitudinal sizes (corresponding to a minimal increment of longitudinal heating) depend on it too. (iii) The additional increase of the longitudinal emittance in comparison with the linear model prediction (which was detected in [17]) has been described in more detail. The statistical approach gives cogent evidence of the efficiency of the head-tail rotation procedure for suppression of nonparaxiality related effects. (iv) It seems that if symplectic emittance exchange parameters will have reasonable values, this scheme can be included to the general scenario starting from the first exchange from horizontal $(x)$ to longitudinal degree of freedom $2 \rightarrow 2^{\prime}$ (marked by the blue circle marker in Fig. 10).

\section{ACKNOWLEDGMENTS}

We are grateful to S. I. Eidelman, V. V. Parkhomchuk, E. A. Perevedentsev, and N. A. Vinokurov for useful discussions. Also we wish to acknowledge the help of P. $\mathrm{McC}$ all and A. Burov for proof reading the manuscript.

\section{APPENDIX A: LINEARIZED MODEL OF TRANSVERSE MOTION}

The transverse motion can be approximately described by a paraxial linear model with a fixed mean energy of a beam; in reality it conforms to short enough sections with matter which alternate with sections of energy recovery. For the case of paraxial motion, it is simple to replace time derivatives to those over the longitudinal path, $s$, in the equation of motion. Using notations in which operators $\frac{d}{d t}$ and $\frac{d}{d s}$ are denoted as ' and ', respectively, and neglecting a variation of the total mean velocity (which is applicable in this approach),

$$
\begin{aligned}
\frac{d p_{x}}{d t} & =\gamma_{\mu} m_{\mu} \frac{d}{d t}(\frac{d x}{d s} \underbrace{\frac{d s}{d t}}_{=v_{\mu}}) \\
& =\gamma_{\mu} m_{\mu}[v_{\mu} \frac{d}{d t}\left(\frac{d x}{d s}\right)+x^{\prime} \underbrace{\frac{d}{d t}}_{\approx 0} v_{\mu}] \\
& =\gamma_{\mu} m_{\mu} v_{\mu} \frac{d x^{\prime}}{d s} \frac{d s}{d t}=\gamma_{\mu} \beta_{\mu}^{2} m_{\mu} c^{2} x^{\prime \prime} .
\end{aligned}
$$

The frictional force

$$
F^{\mathrm{fr}}{ }_{x} \approx F^{\mathrm{fr}} x^{\prime}=-(d E / d l) x^{\prime}
$$

and focusing force

$$
F^{\mathrm{foc}}=-e \beta_{\mu} G x,
$$

where $G$ is a field gradient, substituted into the equation of motion

$$
\frac{d \mathbf{p}}{d t}=\sum_{i} \mathbf{F}_{i}
$$

without taking into account scattering, immediately result in a standard equation for damped oscillations for one of the transverse degrees of freedom:

$$
x^{\prime \prime}+2 \lambda_{s} x^{\prime}+k_{s}^{2} x=0,
$$

where $\lambda_{s}$ and $k_{s}$ are determined by

$$
\lambda_{s}=\frac{1}{2} \frac{1}{\gamma_{\mu} \beta_{\mu}^{2} m_{\mu} c^{2}} \frac{d E}{d l}, \quad k_{s}=\sqrt{\frac{e G}{\gamma_{\mu} \beta_{\mu} m_{\mu} c^{2}}} .
$$

The $d E / d l$ value of average friction forces due to ionization losses is characterized by the Bethe-Bloch equation [22]:

$-\frac{d E}{d l}\left(\beta_{\mu}\right)=\frac{4 \pi n_{\mathrm{e}} e^{4}}{\beta_{\mu}^{2} m_{\mathrm{e}} c^{2}}\left(\ln \frac{\beta_{\mu} \gamma_{\mu} \sqrt{2 m_{\mathrm{e}} c^{2} T_{\max }}}{\bar{I}}-\beta_{\mu}^{2}\right)$,

where $n_{\mathrm{e}}$ is an electron density of matter, $\bar{I}$ is a mean excitation energy, and $T_{\max }$ is a maximal possible kinetic energy transferred to an electron in a single collision: 


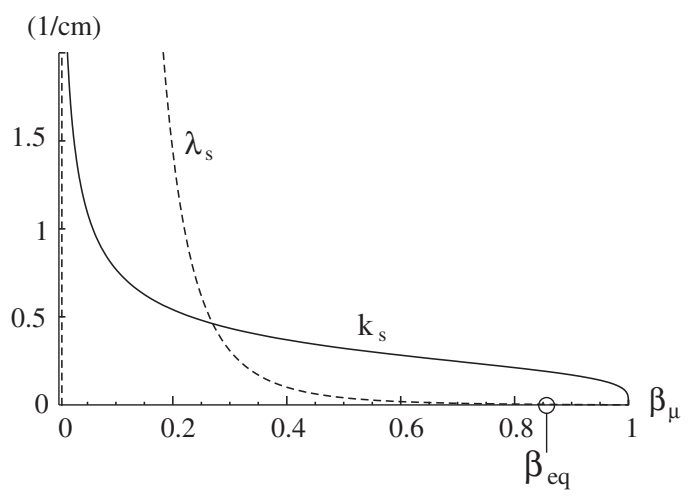

FIG. 11. Dependence of $\lambda_{s}$ and $k_{s}$ on the value of relativistic beta of muons, $\beta_{\mu}$, in Li-rod with a field on its surface of $200 \mathrm{kG}$ and the diameter of $2 \mathrm{~cm}$.

$$
T_{\max }=\frac{2 \beta_{\mu}^{2} \gamma_{\mu}^{2} m_{\mathrm{e}} c^{2}}{1+2 \gamma_{\mu}\left(m_{\mathrm{e}} / m_{\mu}\right)+\left(m_{\mathrm{e}} / m_{\mu}\right)^{2}} .
$$

The behavior of single particle motion crucially depends on the relationship between $\lambda_{s}$ and $k_{s}$. When $\lambda_{s}>k_{s}$ the system is said to be overdamped and the solution decays exponentially without oscillation. If $k_{s}>\lambda_{s}>0$, the system oscillates at reduced frequency (compared to the undamped case when $\lambda_{s}=0$ ) with progressively decaying amplitude. A numerical example of the dependence of $\lambda_{s}$ and $k_{s}$ on the value of muon energy is presented in Fig. 11 and shows that $\lambda_{s}<k_{s}$ for considering energy range.

Averaging over the ensemble of particles makes it possible to rewrite Eq. (A2) as a system of linear differential equations in terms of beam second moments:

$$
\begin{aligned}
\left\langle x^{2}\right\rangle^{\prime} & =2\left\langle x x^{\prime}\right\rangle, \\
\left\langle x x^{\prime}\right\rangle^{\prime} & =\left\langle x^{\prime} x^{\prime}\right\rangle+\left\langle x x^{\prime \prime}\right\rangle=\left\langle x^{\prime 2}\right\rangle-k_{s}^{2}\left\langle x^{2}\right\rangle-2 \lambda_{s}\left\langle x x^{\prime}\right\rangle, \\
\left\langle x^{\prime 2}\right\rangle^{\prime} & =2\left\langle x^{\prime} x^{\prime \prime}\right\rangle=-2 k_{s}^{2}\left\langle x x^{\prime}\right\rangle-4 \lambda_{s}\left\langle x^{\prime 2}\right\rangle .
\end{aligned}
$$

Converting it to a matrix form and adding the column corresponding to the scattering process omitted in Eq. (A2), we have

$$
\left[\begin{array}{c}
\left\langle x^{2}\right\rangle \\
\left\langle x x^{\prime}\right\rangle \\
\left\langle x^{\prime 2}\right\rangle
\end{array}\right]^{\prime}=\left[\begin{array}{ccc}
0 & 2 & 0 \\
-k_{s}^{2} & -2 \lambda_{s} & 1 \\
0 & -2 k_{s}^{2} & -4 \lambda_{s}
\end{array}\right]\left[\begin{array}{c}
\left\langle x^{2}\right\rangle \\
\left\langle x x^{\prime}\right\rangle \\
\left\langle x^{\prime 2}\right\rangle
\end{array}\right]+\left[\begin{array}{c}
0 \\
0 \\
\Theta_{x}^{2}
\end{array}\right] .
$$

The two zeros in the last column describe a zero value of the average scattering angle and a fact that the scattering process does not produce immediate influence on a transverse coordinate. The value of $\Theta_{x}$ has a meaning of a rootmean-square scattering angle per unit of length passed in matter, which in the first approximation can be described by the expression for multiple Coulomb scattering [23]:

$$
\Theta_{x}^{2}=\frac{4 \pi n_{\mathrm{e}} z^{2}(Z+1) e^{4}}{\gamma_{\mu}^{2} \beta_{\mu}^{4} m_{\mu}^{2} c^{4}} L_{\mathrm{C}},
$$

where $z$ and $Z$ are charges of a scattered particle and scattering one, respectively (in elementary electron charge) and $L_{\mathrm{C}}$ is a Coulomb logarithm.

A solution of this equation is given by a sum of the general solution

$$
\begin{aligned}
\left\langle x^{2}\right\rangle_{\mathrm{GS}}= & \exp \left(-2 \lambda_{s} s\right)\left[C_{1} \frac{1}{k_{s}^{2}}\right. \\
& +C_{2}\left(\frac{\lambda_{s}^{2}-\omega^{2}}{k_{s}^{4}} \cos 2 \omega s-\frac{2 \lambda_{s} \omega}{k_{s}^{4}} \sin 2 \omega s\right) \\
& \left.+C_{3}\left(\frac{\lambda_{s}^{2}-\omega^{2}}{k_{s}^{4}} \sin 2 \omega s+\frac{2 \lambda_{s} \omega}{k_{s}^{4}} \cos 2 \omega s\right)\right], \\
\left\langle x x^{\prime}\right\rangle_{\mathrm{GS}}= & \exp \left(-2 \lambda_{s} s\right)\left[-C_{1} \frac{\lambda_{s}}{k_{s}^{2}}\right. \\
& +C_{2}\left(-\frac{\lambda_{s}}{k_{s}^{2}} \cos 2 \omega s+\frac{\omega}{k_{s}^{2}} \sin 2 \omega s\right) \\
& \left.+C_{3}\left(-\frac{\lambda_{s}}{k_{s}^{2}} \sin 2 \omega s-\frac{\omega}{k_{s}^{2}} \cos 2 \omega s\right)\right], \\
\left\langle x^{\prime 2}\right\rangle_{\mathrm{GS}}= & \exp \left(-2 \lambda_{s} s\right)\left[C_{1}+C_{2} \cos 2 \omega s+C_{3} \sin 2 \omega s\right],
\end{aligned}
$$

where $\omega \equiv \operatorname{def} \sqrt{k_{s}^{2}-\lambda_{s}^{2}}$, and a partial one

$$
\begin{aligned}
\left\langle x^{2}\right\rangle_{\mathrm{PS}} & =\frac{\Theta_{x}^{2}}{4 \lambda_{s} k_{s}^{2}}, \\
\left\langle x x^{\prime}\right\rangle_{\mathrm{PS}} & =0, \\
\left\langle x^{\prime 2}\right\rangle_{\mathrm{PS}} & =\frac{\Theta_{x}^{2}}{4 \lambda_{s}} .
\end{aligned}
$$

A substitution of the initial conditions $\left(\left\langle x^{2}\right\rangle_{0},\left\langle x x^{\prime}\right\rangle_{0},\left\langle x^{\prime 2}\right\rangle_{0}\right)$ into a complete solution with $s=0$ determines arbitrary constants $C_{i}$ :

$$
\begin{aligned}
{\left[\begin{array}{l}
C_{1} \\
C_{2} \\
C_{3}
\end{array}\right]=} & \frac{1}{2 \omega^{2}}\left[\begin{array}{ccc}
k_{s}^{4} & 2 \lambda_{s} k_{s}^{2} & k_{s}^{2} \\
-k_{s}^{4} & -2 \lambda_{s} k_{s}^{2} & \left(\omega^{2}-\lambda_{s}^{2}\right) \\
0 & -2 \omega k_{s}^{2} & -2 \omega \lambda_{s}
\end{array}\right]\left[\begin{array}{c}
\left\langle x^{2}\right\rangle_{0} \\
\left\langle x x^{\prime}\right\rangle_{0} \\
\left\langle x^{\prime 2}\right\rangle_{0}
\end{array}\right] \\
& +\frac{1}{2 \omega^{2}} \frac{\Theta_{x}^{2}}{2 \lambda_{s}}\left[\begin{array}{c}
-k_{s}^{2} \\
\lambda_{s}^{2} \\
\omega \lambda_{s}
\end{array}\right] .
\end{aligned}
$$

From this expression, we can obtain a nonoscillatory solution for beam second moments as well as matched initial conditions for it by making $C_{2}$ and $C_{3}$ equal to zero:

$$
\begin{aligned}
\left\langle x^{2}\right\rangle^{M} & =\frac{1}{\lambda_{s}}\left(\lambda_{s}\left\langle x^{2}\right\rangle_{0}-\frac{\Theta_{x}^{2}}{4 k_{s}^{2}}\right) e^{-2 \lambda_{s} s}+\frac{\Theta_{x}^{2}}{4 \lambda_{s} k_{s}^{2}}, \\
\left\langle x x^{\prime}\right\rangle^{M} & =-\left(\lambda_{s}\left\langle x^{2}\right\rangle_{0}-\frac{\Theta_{x}^{2}}{4 k_{s}^{2}}\right) e^{-2 \lambda_{s} s}, \\
\left\langle x^{\prime^{2}}\right\rangle^{M} & =\frac{k_{s}^{2}}{\lambda_{s}}\left(\lambda_{s}\left\langle x^{2}\right\rangle_{0}-\frac{\Theta_{x}^{2}}{4 k_{s}^{2}}\right) e^{-2 \lambda_{s} s}+\frac{\Theta_{x}^{2}}{4 \lambda_{s}}
\end{aligned}
$$

and 


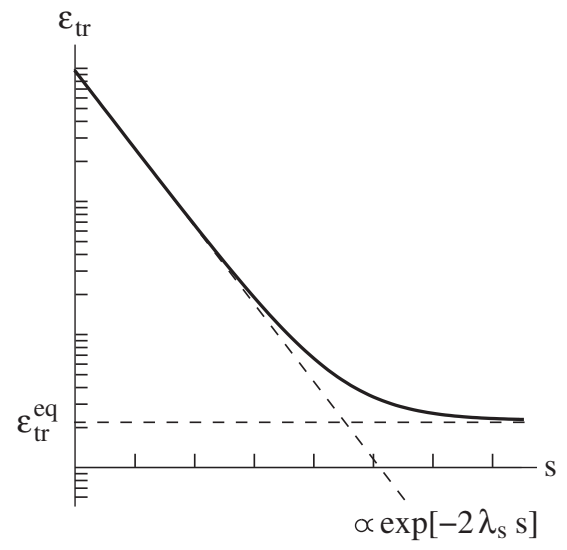

FIG. 12. Behavior of the transverse emittance in logarithmic scale.

respectively.

$$
\begin{aligned}
& \left\langle x x^{\prime}\right\rangle_{0}^{M}=\frac{\Theta_{x}^{2}-4 \lambda_{s} k_{s}^{2}\left\langle x^{2}\right\rangle_{0}}{4 k_{s}^{2}}, \\
& \left\langle x^{\prime 2}\right\rangle_{0}^{M}=k_{s}^{2}\left\langle x^{2}\right\rangle_{0},
\end{aligned}
$$

It seems that asymptotics of the complete solution is a partial solution [Eq. (A4)] because of the presence in the general solution of the exponentially damped factor. This asymptotics also gives the equilibrium value of the transverse emittance:

$$
\varepsilon_{\mathrm{tr}}^{\mathrm{eq}}=\lim _{s \rightarrow \infty} \sqrt{\left\langle x^{2}\right\rangle_{\mathrm{PS}}\left\langle x^{\prime 2}\right\rangle_{\mathrm{PS}}-\left\langle x x^{\prime}\right\rangle_{\mathrm{PS}}^{2}}=\frac{\Theta_{x}^{2}}{4 \lambda_{s} k_{s}} .
$$

Note that, due to the presence of a diffusion (multiple scattering), the cooling decrement is no longer constant. In the limit of a large transverse emittance (compared to $\varepsilon_{\text {tr }}^{\mathrm{eq}}$ ), the value of $\varepsilon_{\text {tr }}$ cools down $\propto e^{-2 \lambda_{s} s}$, but as a system approaches equilibrium the decrement of cooling becomes smaller and tends to zero as $s$ tends to infinity (see Fig. 12).

\section{APPENDIX B: LINEARIZED MODEL OF LONGITUDINAL MOTION}

Similarly to the model selected for the transverse motion, in a paraxial case one can describe the longitudinal motion of a test particle relative to the equilibrium one with an average beam energy. The time derivative of momentum in the case when an acting force is directed along velocity

$$
\frac{d p}{d t}=m_{\mu} \gamma_{\mu}^{3} \dot{v}
$$

substituted into Eq. (A1), gives a differential equation of the second order (Newton equation):

$$
\ddot{z}(t)=\frac{1}{m_{\mu} \gamma_{\mu}^{3}} \sum_{i} F_{i} .
$$

In contrast to Appendix A, for the longitudinal motion there is no focusing force when particles move in matter, which appears only in the section of energy recovery. A full friction force can be represented as a sum of average losses $F^{\text {fr }}$ [Eq. (A3)] and stochastic force $f$ responsible for fluctuations of average losses:

$$
\ddot{z}(t)=\frac{1}{m_{\mu} \gamma_{\mu}^{3}}\left(-\frac{d E}{d l}-f(t)\right) .
$$

Having selected an equilibrium particle which moves with average velocity of a beam $\beta_{\text {eq }}$, and linearizing $d E / d l$ as $\kappa \beta_{\mu}+b$ (Fig. 13), one can obtain the equation of motion for the equilibrium particle:

$$
\ddot{z}_{\mathrm{eq}}=2 \lambda_{t} \dot{\mathrm{z}}_{\mathrm{eq}}-\frac{b}{m_{\mu} \gamma_{\mu}^{3}},
$$

where

$$
\lambda_{t}=-\frac{1}{2} \frac{\kappa c}{\gamma_{\mu}^{3} m_{\mu} c^{2}} .
$$

The set of Eqs. (B1) and (B2) gives the equation for a deviation of a test particle from the equilibrium one:

$$
\left(\ddot{z}-\ddot{z}_{\mathrm{eq}}\right)=2 \lambda_{t}\left(\dot{z}-\dot{z}_{\mathrm{eq}}\right)-f(t) .
$$

Turning it to a differential equation of the first order for a relative velocity, one obtains

$$
\Delta \dot{v}=2 \lambda_{t} \Delta v-f(t),
$$

which solution is

$$
\Delta v(t)=\Delta v_{0} e^{2 \lambda_{t} t}-e^{2 \lambda_{t} t} \int_{0}^{t} e^{-2 \lambda_{t} \tau} f(\tau) d \tau
$$

where $\Delta v_{0}=\left.\Delta v\right|_{t=0}$ is the initial condition.

Considering the fluctuation of energy losses as a Gaussian stochastic process delta correlated in time with a zero mean value [18],

$$
\langle f(t)\rangle=0, \quad\left\langle f\left(t_{1}\right) f\left(t_{2}\right)\right\rangle=B\left(t_{1}, t_{2}\right)=B \delta\left(t_{1}-t_{2}\right),
$$

and averaging over a stochastic force of Eq. (B3) allows one to find the correlation function of velocity:

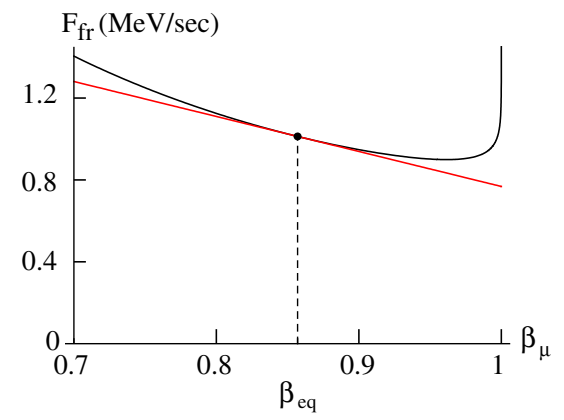

FIG. 13. The average ionization losses (black curve) and its linear approximation (red line) as a function of muon velocity. 

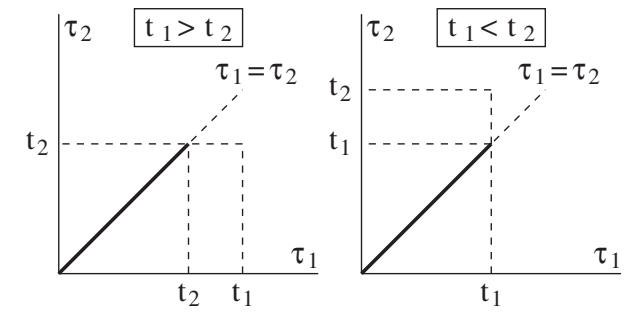

FIG. 14. Domain of integration (thick black line) of $\int_{0}^{t_{1}} d \tau_{1} \int_{0}^{t_{2}} d \tau_{2}\left\{e^{-2 \lambda_{t} \tau_{1}-2 \lambda_{t} \tau_{2}}\left\langle f\left(\tau_{1}\right) f\left(\tau_{2}\right)\right\rangle\right\}$ depending on the relationship between $t_{1}$ and $t_{2}$.

$$
\begin{aligned}
\left\langle\Delta v\left(t_{1}\right) \Delta v\left(t_{2}\right)\right\rangle_{f} \\
=\Delta v_{0}^{2} e^{2 \lambda_{t} t_{1}+2 \lambda_{t} t_{2}}-\Delta v_{0} e^{2 \lambda_{t} t_{1}} \int_{0}^{t_{2}} e^{-2 \lambda_{t} \tau_{2}}\left\langle f\left(\tau_{2}\right)\right\rangle d \tau_{2} \\
\quad-\Delta v_{0} e^{2 \lambda_{t} t_{2}} \int_{0}^{t_{1}} e^{-2 \lambda_{t} \tau_{1}}\left\langle f\left(\tau_{1}\right)\right\rangle d \tau_{1}+e^{2 \lambda_{t} t_{1}+2 \lambda_{t} t_{2}} \\
\quad \times \int_{0}^{t_{1}} d \tau_{1} \int_{0}^{t_{2}} d \tau_{2}\left\{e^{-2 \lambda_{t} \tau_{1}-2 \lambda_{t} \tau_{2}}\left\langle f\left(\tau_{1}\right) f\left(\tau_{2}\right)\right\rangle\right\} .
\end{aligned}
$$

The last integral in this equation depends on the relationship between $t_{1}$ and $t_{2}$ due to causality (see explanatory Fig. 14), which gives

$$
\begin{aligned}
\left\langle\Delta v\left(t_{1}\right) \Delta v\left(t_{2}\right)\right\rangle_{f}= & \left(\Delta v_{0}^{2}+\frac{B}{4 \lambda_{t}}\right) e^{2 \lambda_{t}\left(t_{1}+t_{2}\right)} \\
& -\frac{B}{4 \lambda_{t}} e^{2 \lambda_{t}\left(t_{1}+t_{2}\right)-4 \lambda_{t} \min \left(t_{1}, t_{2}\right)} .
\end{aligned}
$$

Assuming $t_{1}=t_{2}$ in it gives the root-mean-square velocity spread:

$$
\left\langle\Delta v^{2}(t)\right\rangle_{f}=\left(\Delta v_{0}^{2}+\frac{B}{4 \lambda_{t}}\right) e^{4 \lambda_{t} t}-\frac{B}{4 \lambda_{t}} .
$$

Comparison of Eqs. (B4) and (A5c) shows the meaning of $B$-this is a root-mean-square velocity "spread" per unit time, arising because of fluctuations of energy losses:

$$
\Theta_{d E / E}^{2}=2 \pi \beta_{\mu} c r_{\mu} n_{e}\left[2-\beta_{\mu}^{2}\right]
$$

from which

$$
\Theta_{v} \equiv{ }^{\text {def }} B^{1 / 2} \approx \frac{c}{\beta_{\mu} \gamma_{\mu}^{2}} \Theta_{d E / E} .
$$

Note that, besides a difference in a sign of $\lambda_{t}$ and $\lambda_{s}$ in Eqs. (B4) and (A5c), there is also a factor-of-two difference in exponent. This fact is a direct consequence of the presence of a focusing force for the case of transverse motion: the average of friction force squared is twice smaller because the friction force acting on a particle varies along with $x^{\prime}$ as the sine function. Adding longitudinal rf and integrating over synchrotron oscillations gives a focusing force which removes this distinction.

Making a similar proof for the value of the longitudinal deviation $\Delta z(t)$ : writing a solution in an integrated form from $\Delta \dot{z}=\Delta v$

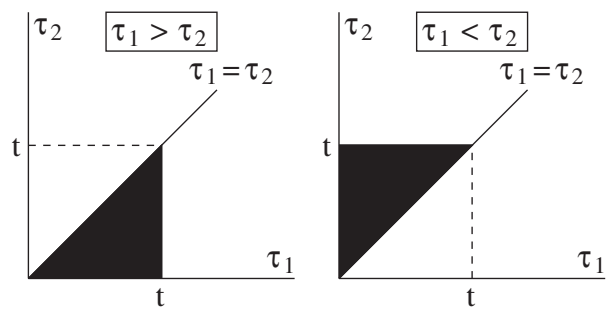

FIG. 15. Domain of integration (ם) of $\int_{0}^{t} d \tau_{1} \times$ $\int_{0}^{t} d \tau_{2}\left\{\left\langle\Delta v\left(\tau_{1}\right) \Delta v\left(\tau_{2}\right)\right\rangle_{f}\right\}$ for $\tau_{1}>\tau_{2}$ and $\tau_{1}<\tau_{2}$.

$$
\Delta z(t)=\Delta z_{0}+\int_{0}^{t} \Delta v(\tau) d \tau
$$

and averaging it by a stochastic force, one can find the rootmean-square value of $\Delta z(t)$ :

$$
\begin{aligned}
\left\langle\Delta z^{2}(t)\right\rangle_{f}= & \Delta z_{0}^{2}+2 \Delta z_{0} \int_{0}^{t}\langle\Delta v(\tau)\rangle_{f} d \tau \\
& +\int_{0}^{t} d \tau_{1} \int_{0}^{t} d \tau_{2}\left\{\left\langle\Delta v\left(\tau_{1}\right) \Delta v\left(\tau_{2}\right)\right\rangle_{f}\right\}
\end{aligned}
$$

To perform the last integral, one needs to split the domain of integration (see explanatory Fig. 15), which finally gives

$$
\begin{aligned}
\left\langle\Delta z^{2}(t)\right\rangle_{f}= & \Delta z_{0}^{2}+\left(2 \Delta z_{0} \Delta v_{0}-\frac{\Theta_{v}^{2}}{4 \lambda_{t}^{2}}\right)\left(\frac{e^{2 \lambda_{t} t}-1}{2 \lambda_{t}}\right) \\
& +\left(\Delta v_{0}^{2}+\frac{\Theta_{v}^{2}}{4 \lambda_{t}}\right)\left(\frac{e^{2 \lambda_{t} t}-1}{2 \lambda_{t}}\right)^{2}+\frac{\Theta_{v}^{2}}{4 \lambda_{t}^{2}} t
\end{aligned}
$$

The calculation of the cross correlation function gives

$$
\begin{aligned}
\langle\Delta z(t) \Delta v(t)\rangle_{f}= & {\left[\left(\Delta v_{0}^{2}+\frac{\Theta_{v}^{2}}{4 \lambda_{t}}\right) e^{2 \lambda_{t} t}-\frac{\Theta_{v}^{2}}{4 \lambda_{t}}\right] \frac{e^{2 \lambda_{t} t}-1}{2 \lambda_{t}} } \\
& +\Delta z_{0} \Delta v_{0} e^{2 \lambda_{t} t}
\end{aligned}
$$

Finally, averaging over the initial ensemble of particles and turning to variables of energy deviation and arrival time (here and further multiplied by the speed of light)

$$
\frac{\Delta E}{E_{\mathrm{eq}}} \approx \beta_{\mathrm{eq}} \gamma_{\mathrm{eq}}^{2} \frac{\Delta v}{c}, \quad c \Delta t \approx \beta_{\mathrm{eq}}^{-1} \Delta z
$$

one can find an expression describing the behavior of the longitudinal emittance for a beam moving in matter: 


$$
\begin{aligned}
\varepsilon_{\text {long }}= & \sqrt{\left\langle(c \Delta t)^{2}\right\rangle\left\langle\left(\frac{\Delta E}{E_{\mathrm{eq}}}\right)^{2}\right\rangle-\left\langle(c \Delta t)\left(\frac{\Delta E}{E_{\mathrm{eq}}}\right)\right\rangle^{2},} \\
\left\langle\left(\frac{\Delta E}{E_{\mathrm{eq}}}\right)^{2}\right\rangle= & \left(\Delta^{2}+\frac{\Theta_{d E / E}^{2}}{4 \lambda_{t}}\right) e^{4 \lambda_{t} t}-\frac{\Theta_{d E / E}^{2}}{4 \lambda_{t}}, \\
\left\langle(c \Delta t)\left(\frac{\Delta E}{E_{\mathrm{eq}}}\right)\right\rangle= & {\left[\left(\Delta^{2}+\frac{\Theta_{d E / E}^{2}}{4 \lambda_{t}}\right) e^{2 \lambda_{t} t}-\frac{\Theta_{d E / E}^{2}}{4 \lambda_{t}}\right] } \\
& \times\left(\frac{c}{\beta_{\mathrm{eq}}^{2} \gamma_{\mathrm{eq}}^{2}} \frac{e^{2 \lambda_{t} t}-1}{2 \lambda_{t}}\right)+\zeta e^{2 \lambda_{t} t}, \\
\left\langle(c \Delta t)^{2}\right\rangle= & \left(\Delta^{2}+\frac{\Theta_{d E / E}^{2}}{4 \lambda_{t}}\right)\left(\frac{c}{\beta_{\mathrm{eq}}^{2} \gamma_{\mathrm{eq}}^{2}} \frac{e^{2 \lambda_{t} t}-1}{2 \lambda_{t}}\right)^{2} \\
& +\left(2 \zeta-\frac{1}{\lambda_{t}} \frac{c}{\beta_{\mathrm{eq}}^{2} \gamma_{\mathrm{eq}}^{2}} \frac{\Theta_{d E / E}^{2}}{4 \lambda_{t}}\right) \\
& \times\left(\frac{c}{\beta_{\mathrm{eq}}^{2} \gamma_{\mathrm{eq}}^{2}} \frac{e^{2 \lambda_{t} t}-1}{2 \lambda_{t}}\right) \\
& +\frac{c^{2}}{\beta_{\mathrm{eq}}^{4} \gamma_{\mathrm{eq}}^{4}} \frac{1}{\lambda_{t}^{2}} \frac{\Theta_{d E / E}^{2}}{4 \lambda_{t}} \lambda_{t} t+\delta^{2},
\end{aligned}
$$

where

$$
\delta \equiv \operatorname{def}\left\langle(c \Delta t)^{2}\right\rangle_{0}^{1 / 2}, \quad \Delta \equiv \operatorname{def}\left\langle\left(\frac{\Delta E}{E_{\text {eq }}}\right)^{2}\right\rangle_{0}^{1 / 2}
$$

and

$$
\zeta \equiv \operatorname{def}\left\langle(c \Delta t)\left(\frac{\Delta E}{E_{\mathrm{eq}}}\right)\right\rangle_{0}
$$

are initial data averaged over the initial ensemble. The large time asymptotics of the longitudinal emittance is

$$
\lim _{t \rightarrow \infty} \varepsilon_{\text {long }}=\frac{c}{\beta_{\text {eq }}^{2} \gamma_{\mathrm{eq}}^{2}} \sqrt{\left(\Delta^{2}+\frac{\Theta_{d E / E}^{2}}{4 \lambda_{t}}\right) \frac{\Theta_{d E / E}^{2}}{4 \lambda_{t}}} \frac{\left(\lambda_{t} t\right)^{1 / 2} e^{2 \lambda_{t} t}}{\lambda_{t}} .
$$

[1] G.I. Budker, in 7th International Conference on HighEnergy Accelerators of Charged Particles, YerevanTsaghkadzor, USSR, 1969, edited by A.I. Alikhanian (Izd-vo Akademii Nauk Armianskoi SSR, Erevan, 1970), Vol. 1, pp. 33-39; D. B. Cline, in Physics Potential and Development of $\mu^{+} \mu^{-}$Colliders, Second Workshop: Sausalito, CA, 1994, AIP Conf. Proc. No. 352, edited by D. B. Cline (AIP Press, New York, 1996), p. 4.

[2] G. I. Budker, in 15th International Conference on High Energy Physics, 1970, Kiev, USSR (1971), pp. 10171023; D. B. Cline, in Physics Potential and Development of $\mu^{+} \mu^{-}$Colliders, Second Workshop: Sausalito, CA, 1994 (Ref. [1]), pp. 4-5.

[3] A. N. Skrinsky, in the International Seminar on Prospects of High-Energy Physics, Morges, Switzerland, 1971; D. B. Cline, in Physics Potential and Development of $\mu^{+} \mu^{-}$ Colliders, Second Workshop: Sausalito, CA, 1994 (Ref. [1]), p. 6.
[4] A. A. Kolomenskii, Sov. At. Energy 19, 1511 (1965).

[5] D. Neuffer, Part. Accel. 14, 75 (1983).

[6] Yu. M. Ado and V. I. Balbekov, Sov. At. Energy 31, 731 (1971).

[7] A. N. Skrinsky and V. V. Parkhomchuk, Sov. J. Part. Nucl. 12, 223 (1981), in Russian.

[8] V. Shiltsev, Mod. Phys. Lett. A 25, 567 (2010).

[9] M.S. Zisman, in Proceedings of the 1st North American Particle Accelerator Conference, New York, NY, 2011 (IEEE, Piscataway, NJ, 2011), THOBN1.

[10] Y. Torun, D. Huang, J. Norem, R. B. Palmer, A. D. Bross, M. Chung, A. Jansson, A. Moretti, K. Yonehara, D. Li, and R. A. Rimmer, in contributions to the Proceedings of 1st International Particle Accelerator Conference: IPAC'10, Kyoto, 2010 (IPAC'10 OC/ACFA, 2010), pp. 3780-3782, THPEA046 [http://accelconf.web.cern.ch/AccelConf/ IPAC10/papers/thpea054.pdf].

[11] L. Coney, in Proceedings of the 23rd Particle Accelerator Conference, Vancouver, Canada, 2009 (IEEE, Piscataway, NJ, 2009), pp. 1680-1682, TU6RFP057; D. Li, in Proceedings of COOL 2009: Workshop on Beam Cooling and Related Topics, 2009, Lanzhou, China (2009), pp. 71-75, TUA1MCIO01 [http://epaper.kek.jp/ COOL2009/papers/tua1mcio01.pdf ].

[12] A. Mikhailichenko, Cornell University Colliding Beam Notes No. CBN 103, 2010.

[13] V. Lebedev, "Ionization Cooling with Lithium Lenses," Muon Collider Design Workshop, 2009, Brookhaven National Laboratory (2009).

[14] V. Lebedev, Technical Report No. FERMILAB-PBARNOTE-666, Fermilab, 2001.

[15] T. Zolkin and A. N. Skrinsky, in Proceedings of the 1st North American Particle Accelerator Conference, New York, NY, 2011 (IEEE, Piscataway, NJ, 2011), MOP062.

[16] A. N. Skrinsky, in Beam Dynamics and Technology Issues for $\mu^{+} \mu^{-}$Colliders: 9th Advanced ICFA Beam Dynamics Workshop, Mon-tauk, NY, 1995, AIP Conf. Proc. No. 372, edited by Juan C. Gallardo (American Institute of Physics, New York, 1996), pp. 133-139; in Proceedings of the Eleventh International Advanced ICFA Beam Dynamic Workshop on Beam Cooling and Instability Damping Dedicated to the 30th Anniversary of Electron Cooling on board a ship from Moscow to Nizhny Novgorod [Nucl. Instrum. Methods Phys. Res., Sect. A 391, 188 (1997)].

[17] A. N. Skrinsky and T. Zolkin, Nucl. Instrum. Methods Phys. Res., Sect. A 608, 42 (2009).

[18] C. Grupen, B.A. Shwartz, and H. Spieler, Particle Detectors (Cambridge University Press, Cambridge, England, 2008), Chap. 1, pp. 3-12.

[19] C. Wang and K.J. Kim, Phys. Rev. Lett. 88, 184801 (2002).

[20] M. Cornacchia and P. Emma, Phys. Rev. ST Accel. Beams 5, 084001 (2002).

[21] N. Yampolsky, B. Carlsten, R. Ryne, K. Bishofberger, S. Russell, and A. Dragt, arXiv:1010.1558.

[22] W.-M. Yao et al., J. Phys. G 33, 258 (2006).

[23] K. N. Mukhin, Experimental Nuclear Physics: Book 1: Physics of Atomic Nucleu (Energoatomizdat, Moscow, 1993), 5th ed., Chap. 4, pp. 326-330. 